Review Article

\title{
Posttransplant Lymphoproliferative Disorder after Cardiac Transplantation in Children: Life Threatening Complications Associated with Chemotherapy Combined with Rituximab
}

\author{
Norihide Fukushima \\ Department of Therapeutics Strategies for End Organ Dysfunction, Osaka University Graduate School of Medicine, Japan \\ Correspondence should be addressed to Norihide Fukushima; nori@surg1.med.osaka-u.ac.jp
}

Received 16 April 2013; Accepted 15 May 2013

Academic Editors: D. Caselli, H.-J. Kim, and C. Marchese

Copyright (C) 2013 Norihide Fukushima. This is an open access article distributed under the Creative Commons Attribution License, which permits unrestricted use, distribution, and reproduction in any medium, provided the original work is properly cited.

Despite the excellent long-term survival currently achieved in pediatric heart transplant recipients, posttransplant lymphoproliferative disorders (PTLDs) are one of the most important causes of morbidity and mortality after heart transplantation (HTx), especially in children. Timely and accurate diagnosis based on histological examination of biopsy tissue is essential for early intervention for PTLD. Chemotherapy is indicated for patients with poor response to reduction of immunosuppressive medication and for highly aggressive monomorphic PTLD. The use of rituximab in combination with chemotherapy is effective to suppress B cell type PTLD (B-PTLD). However, PTLD relapses frequently and the outcome is still poor. Although everolimus (EVL) has been reported to inhibit growth of human Epstein-Barr-virus- (EBV-) transformed B lymphocytes in vitro and in vivo, EVL has several side effects, such as delayed wound healing and an increase in bacterial infection. During combined treatment of chemotherapy and rituximab, B-PTLDs are sometimes associated with life-threatening complications, such as intestinal perforation and cardiogenic shock due to cytokine release syndrome. In HTx children especially treated with EVL, stoma should be made to avoid reoperation or sepsis in case of intestinal perforation. In cases with cardiac graft dysfunction possibly due to cytokine release syndrome by chemotherapy with rituximab for PTLD, plasma exchange is effective to restore cardiac function and to rescue the patients.

\section{Introduction}

More than five hundred heart transplants (HTx) in children $(<18$ years age $)$ had been performed every year in the world since 2008 [1]. Despite the excellent long-term survival currently achieved in children who underwent $\mathrm{HTx}$, the immunosuppressive medications required to prevent allograft rejection throughout his or her life may cause significant morbidity and mortality, such as malignancy, renal dysfunction, hypertension, hyperlipidemia, and diabetes mellitus. Although most malignancies in adults are skin cancers [2], almost all malignancies are posttransplant lymphoproliferative disorders (PTLD) in children [1]. PTLD is counted as the cause of death in $2.0 \%, 3.3 \%, 4.1 \%, 8.4 \%$, and $8.2 \%$ of cases in the period from 31 days to 1 year, $<1-3$ years, $<3-5$ years, $<5-10$ years, and $>10$ years after HTx, respectively [1].
PTLD has a wide spectrum of disease manifestations, ranging from a benign plasmacytic hyperplasia and infectious mononucleosis-like PTLD (so called early lesion) to an aggressive monoclonal lymphoma (such as Burkitt lymphoma) that can be fatal [3]. Timely and accurate diagnosis based on histological examination of biopsy tissue is essential for early intervention [3]. Chemotherapy is indicated for patients with poor response to reduction of immunosuppressive medication and for highly aggressive monomorphic PTLD. The use of rituximab in combination with chemotherapy is effective. However, B cell type PTLDs (B-PTLDs) are sometimes associated with life-threatening complications, such as intestinal perforation [4] and cardiogenic shock due to cytokine release syndrome [5-7] after or during chemotherapy combined with rituximab.

In this paper, a representative pediatric case with lifethreatening complications during the treatment of PTLD 
after HTx is described and therapeutic strategies for these complications are discussed.

\section{Representative Case Report}

2.1. Polymorphic B-PTLD (Diffuse Large B-Cell Lymphoma) with Intestinal Perforation and Cardiogenic Shock after Chemotherapy Combined with Rituximab. A 33-month-old boy underwent successful HTx for bilateral ventricular failure after several surgical interventions for congenital complex cardiac anomaly (bilateral pulmonary banding, Rastelli operation, and truncal valve replacement) in July 2011. He received cyclosporine (CsA), mycophenolate mofetil (MMF), and rabbit antithymocyte globulin (RATG) for posttransplant immunosuppression. He was Epstein-Barr-virus(EBV-) seronegative before transplant (antivirus capsid antigen immunoglobulin (VCA-IgG): $<10$-fold) and his donor was EBV seropositive. As he experienced acute cellular rejection by echocardiography in December 2011, CsA was converted to tacrolimus (Tac). As he was a case of everolimus (EVL) study protocol, MMF was converted to EVL at the same time. He remained EBV seronegative in February 2012. Scheduled endomyocardial biopsy obtained in July 2012 revealed no acute rejection in the cardiac graft.

On July 17, 2012, at 45 months of age and 12 months after transplant, he had a high fever and a rash in bilateral hands on the next day. After administration of antibiotics his temperature dropped down, but he had a recurrent high fever on 22th and diarrhea on 25th and was admitted on 30th in Kyushu University Hospital. He received ganciclovir (GCV) and valganciclovir (VGCV) and intravenous immunoglobulin (IVIG) with high titer of antibodies against EBV to prevent the development of PTLD but still had a high fever. His EBV antibody titer for VCA-IgG became positive (160-fold) and EBV titer was increased to $5 \times 10^{3}$ copies $/ 10^{6} \mathrm{WBCs}$ at that time.

Although Tac was converted to MMF, he still had a high fever and was transferred to our hospital on 8th August. A biopsy of the neck lymph node established a diagnosis of polymorphic PTLD, diffuse large B-cell lymphoma (DLBL). Immunoblasts were CD20+, CD79a+, and CD3-. CD3+ cells were also observed. In situ hybridization for EBV early RNA (EBER) showed reactivity in lymphoid cells. Conventional cytogenetic analysis revealed $46, \mathrm{XY}, \mathrm{t}(4 ; 14)(\mathrm{q} 31 ; \mathrm{q} 32)$. At this point, EBV titer was increased to $3.3 \times 10^{4}$ copies $/ 10^{6}$ white blood cell counts (WBCs). EBV antibody titers for VCA-IgG were 320-fold. Laboratory studies showed a white blood cell (WBC) count of $4,390 / \mathrm{mm}^{3}$, hemoglobin $12.5 \mathrm{~g} / \mathrm{dL}$, C-reactive protein (CRP) $9.8 \mathrm{ng} / \mathrm{mL}$, lactate dehydrogenase (LDH) 1,831 IU/L, aspartate transaminase (AST) $77 \mathrm{IU} / \mathrm{L}$, alanine transaminase (ALT) $7 \mathrm{IU} / \mathrm{L}$, urea nitrogen $6 \mathrm{mg} / \mathrm{dL}$, uric acid $3.7 \mathrm{mg} / \mathrm{dL}$, serum creatinine $0.29 \mathrm{mg} / \mathrm{dL}$, soluble interleukin-2 receptor (sIL-2R) level 11,289 U/mL, and brain natriuretic peptide (BNP) $46 \mathrm{pg} / \mathrm{mL}$.

${ }^{18}$ F-fluorodeoxyglucose (FDG) positron emission tomography-computed tomography (PET/CT) scan showed high uptake in almost all small intestine (Figure 1). As metabolic tumor volume estimated by FDG-PET/CT

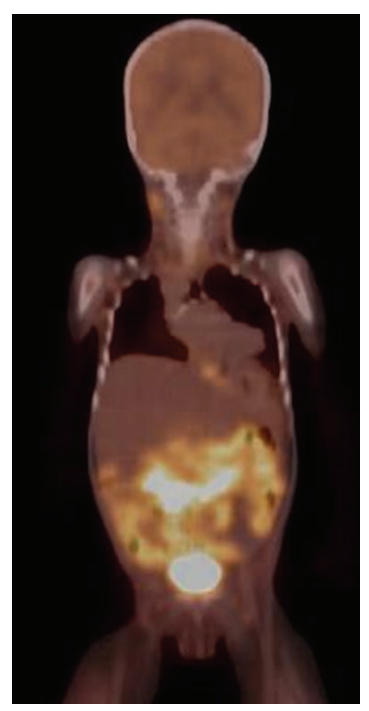

FIGURE 1: ${ }^{18}$ F-fluorodeoxyglucose (FDG) positron emission tomography-CT (PET/CT) in the present case showed high uptake in almost all small intestine. Metabolic tumor volume was estimated to be very high.

was extremely high, a chemotherapy regimen (rituximab combined with cyclophosphamide, adriamycin, vincristine, and prednisone; R-CHOP) for DLBL seemed to be too toxic for him. Therefore, he received rituximab $\left(375 \mathrm{mg} / \mathrm{m}^{2}\right)$ on 13 th and only a half dose of vincristine $(0.5 \mathrm{mg})$ on $18 \mathrm{th}$. However, he had hemodynamic shock, a high fever, and abdominal pain. Abdominal X-ray revealed free air in his peritoneal cavity. He had undergone surgery because of acute abdomen on August 19. At laparotomy, multiple perforations approximately $20 \mathrm{~cm}$ from the Treitz ligament to the ileum end and multiple mesenteric lymph node swellings were observed. As he had received EVL and almost total small intestine were involved, primary closures were performed on several apparent perforated regions and ulcerative lesions and a jejunostomy was made anally $10 \mathrm{~cm}$ from the Treiz ligament.

As he could not take any oral medication, he received anti-IL2R antibody, basiliximab (5 mg), and high dose predonisolone (PRD) (20 mg/day) intravenously. Daily echocardiography revealed progressed bilateral ventricular dysfunction and he required a dobutamine (DOB) infusion in a dose of $5.4 \mathrm{mcg} / \mathrm{kg} / \mathrm{min}$. A left ventricular (LV) ejection fraction (EF) measured on August 21 was 22\%, but no echocardiographic findings suggesting allograft rejection such as increased LV wall thickness or pericardial effusion were observed. Laboratory studies at this time showed a WBC count of $2,820 / \mathrm{mm}^{3}$, hemoglobin $11.0 \mathrm{~g} / \mathrm{dL}, \mathrm{CRP} 26.6 \mathrm{ng} / \mathrm{mL}$, LDH 1,450 IU/L, AST $390 \mathrm{IU} / \mathrm{L}$, ALT $96 \mathrm{IU} / \mathrm{L}$, urea nitrogen $24 \mathrm{mg} / \mathrm{dL}$, uric acid $3.0 \mathrm{mg} / \mathrm{dL}$, serum creatinine $0.54 \mathrm{mg} / \mathrm{dL}$, sIL-2R level 1,943 U/mL, and BNP 2,866 pg/mL. Therefore, we thought that cytokine or toxin release from the apoptotic tumor cells and a higher tumor burden as well as intestinal perforation lead to left ventricular dysfunction resulting in cardiogenic shock. His plasma was replaced by $1,200 \mathrm{~mL}$ of 
fresh frozen plasma (FFP) on 21st, 22nd, and 23rd in order to reduce released cytokines and/or toxins. LVEF rapidly increased to $55 \%$ and DOB infusion was discontinued on 27th.

A drip infusion of cytarabine $\left(150 \mathrm{mg} / \mathrm{m}^{2} /\right.$ day $)$ was given from August 27th to September 2nd. Immunosuppressive therapy with intravenous CsA was started on 4th September to maintain trough levels at $60-80 \mathrm{ng} / \mathrm{mL}$ with intravenous PRD $(20 \mathrm{mg} /$ day $)$. Although rituximab $\left(375 \mathrm{mg} / \mathrm{m}^{2}\right)$ was given on September 7th, no decrease in LVEF was observed probably because tumor volume was already reduced by the first chemotherapy with rituximab and vincristine. Cyclophosphamide was given on 25th. On October 2nd, a dose of CsA was increased to maintain trough levels at 80$120 \mathrm{ng} / \mathrm{mL}$ with reduction in a dose of intravenous PRD $(10 \mathrm{mg} /$ day). He just received final course of chemotherapy and showed no signs of symptoms of remission of PTLD, intestinal perforation or acute allograft rejection 7 months following intestinal perforation and cardiogenic shock due to the first dose of chemotherapy.

\section{Incidence of PTLD}

The incidence of PTLD after solid organ transplantation is markedly different in children as compared to adults and also varies according to the type of organ transplant because of differences in the intensity of immunosuppressive regimens used $[3,9,10]$. In adults, kidney recipients have the lowest frequency of PTLD (1\%-2\%); liver recipients have a slightly higher incidence $(1 \%-3 \%)$, followed by adult heart recipients $(1 \%-6 \%)[1,10-14]$, heart-lung recipients $(2 \%-6 \%)$, and lung recipients (4-10\%) $[1,10,13]$; and small bowel recipients have the highest incidence (up to 20\%) [10]. Children have 2 to 3 -fold incidence of PTLD compared to adults: $1 \%-10 \%$ in kidney transplants; $4 \%-15 \%$ in liver transplants, and 6\%-20\% in lung, heart, and heart-lung transplants [15]. Differences according to transplanted organs are primarily due to the varying level of immunosuppression. The higher incidence of PTLD following lung and small intestine transplantation can be attributed to aggressive immunosuppression and the presence of preexisting lymphoid tissue in these organs, which is transferred to the recipient, increasing the probability of EBV infection $[16,17]$.

PTLD may develop at any time following organ transplantation; however, its risk is highest during the first year. Accordingly, early and late forms can be distinguished, with different therapeutic response and prognosis. The incidence decreases with time after the first year [9]. Early development of PTLD is characteristic for heart and lung transplantation, with nearly half of the cases appearing in the first year. However, only $20 \%$ of PTLD cases have an early manifestation in kidney transplanted patients. This difference is due to higher dose immunosuppression and induction treatment following heart and lung transplantation $[17,18]$.

There was a linear decrease in freedom from malignancy from time of pediatric HTx with $16 \%$ of patients having developed a malignancy by 15 years, according to the report from the International Society for Heart and Lung Transplantation [1]. According to a multi-institutional study [14] of 3170 pediatric primary HTx between 1993 and 2009 at 35 institutions in Pediatric Heart Transplant Study (PHTS), overall freedom from PTLD was $98.5 \%$ at 1 year, $94 \%$ at 5 years and $90 \%$ at 10 years. As the PHTS showed that 147 of 151 reported malignancy events were classified PTLD, almost all malignancies were PTLD in children $[1,14]$. Cumulative prevalence of PTLD in survivors was $1.7 \%, 4.9 \%$, and $7.8 \%$ at 1,5 , and 10 years after HTx, respectively [1]. An early phase of peak risk was recorded at around 6 months, followed by a continuous slow decrease in risk, but without ever reaching zero. In contrast, in hematopoietic stem cell transplantation (HSCT) recipients, the onset of PTLD is earlier: within 6 months (median 70-90 days) [19]. Cumulative prevalence of PTLD in HTx survivors in children (7.8\%) was higher than that in adults $(2.0 \%)$ [1]. The higher incidence of PTLD in pediatric recipients is mainly attributed to the development of primary EBV infection after transplantation.

The PHTS reported that the overall risk for PTLD declined in the most recent transplant era (2001-2009, $P=$ 0.003) [14]. As has been observed in previous publications $[17,20]$, there has been a clear improvement in outcomes over time since 1993. The reasons for the reduction in PTLD are not known and are not explained by this study. Of note, there has been increased use of induction therapy in children in recent years [21], and rejection rates have fallen [20]. However, this has not been accompanied by an increased risk of PTLD. Indeed, this PHTS data showed the opposite. The routine use of viral load monitoring by PCR in the recent era has led many centers to adjust immunosuppression when primary EBV infection is first identified [22, 23]. This may have led to prevention of progression of primary EBV infection to symptomatic disease and PTLD. This hypothesis requires testing in prospective clinical trials.

\section{Risk Factors}

4.1. Recipient Age. Recipient age is a risk factor for PTLD $[1,14]$. Freedom from PTLD was lowest in children (ages 1 to $<10$ years) versus infants ( $<1$ year) and adolescents $(10$ to $<18$ years) with children at highest risk for PTLD with a relative risk of 2.4 compared to infants and 1.7 compared to adolescents [14]. The mechanisms for the age-related effects are not entirely clear. The decreased susceptibility of infants and adolescents relative to young children could be attributed to maternal protection and an increasingly competent immune system, respectively. The potential effect of maternal protection can be clearly seen in the EBV serology as a function of age at the time of transplant. The proportion of infants testing EBV+ (65\%) approaches that of adults, strongly suggesting that the detected EBVspecific antibodies are a result of maternal to fetal transfer. These antibodies have a limited half-life, and therefore it would be expected that these passively transferred protective antibodies would wane over a period of months. This corresponds with increasing susceptibility to PTLD observed in patients transplanted 6-9 months after birth. 
4.2. Allograft Rejection. A higher frequency of transplant rejection is another risk factor for PTLD [10]. This may be related to the increased immunosuppression needed to treat allograft rejection.

4.3. EBV Status and Viral Load. EBV status mismatch between recipient and donor (seronegative recipient with seropositive donor) is associated with the development of PTLD. It has been reported that pretransplant EBVseronegative status was significantly $(P=0.001)$ more frequent in patients with PTLD (6 of 12, 50\%) as compared to those without PTLD (11 of 50, 18\%) [24]. The PHTS also reported that positive donor EBV status was a strong risk factor for PTLD in the seronegative recipients, but that risk magnitude was dependent on recipient age at the time of HTx. Nearly $25 \%$ of EBV seronegative recipients of seropositive donors at ages 4-7 years at HTx developed some form of PTLD [14]. It seems likely that immunological maturation (whether related to antibody, CTL, or other protective immune responses) may play an important role in protection of the older seronegative recipient of the seropositive donor. The apparent protective effect of very young age compared to children suggests that EBV-specific antibodies (including those transferred from the mother) may play a role in the avoidance of PTLD and lends credence to the concept that passive administration of anti-EBV antibody to the recipient could potentially protect against PTLD, though this has not yet been proven in prospective human clinical trials [25].

B-PTLD occurring early after transplantation is often associated with high EBV viral loads in peripheral blood samples and these high viral loads often precede clinical symptoms. Green et al. [26] used a preemptive protocol based on EBV PCR threshold surveillance, which led to a decrease of PTLD incidence from $46 \%$ to $16 \%$ in pediatric intestine recipients. They used a threshold of $\geq 200$ genome copies $/ 10^{5}$ lymphocytes in EBV-seropositive children and $\geq 40$ genome copies $/ 10^{5}$ lymphocytes in pre-transplant EBV-seronegative children. A chronic high viral load state with the presence $>16,000$ genome copies/mL whole blood was reported to be a predictor of de novo or recurrent PTLD after pediatric HTx [27]. Thus, EBV serostatus and monitoring EBV viral load are essential for suspecting PTLD and its early management.

Elevated EBV viral loads without clinical symptoms do not seem to be specific for the development of PTLD; EBV viral loads alone cannot be used to identify individual patients who develop PTLD [28]. It must also be noted that EBV-associated PTLD has been described in patients with low or undetectable EBV viral loads [29]. PTLD arises, to a large part, as a consequence of immunosuppression necessary to prevent allograft rejection.

4.4. Immunosuppressive Regimen. Although an increased risk for PTLD has been reported to be associated with the use of induction therapy, especially a use of mouse anti-CD3 monoclonal antibody (OKT3) [11, 30-32], recent reports described no difference in an incidence of PTLD between induction and no induction group except OKT3 [1]. However, a recent single-center pediatric PTLD study showed that longer duration (and, hence, greater dose) of induction treatment significantly increases the risk of PTLD [33]. As many reports, primarily in pediatric liver transplantation, have shown an increase risk of PTLD developing in patients treated with Tac as the primary immunosuppressant drug as compared to CsA [34-37], an incidence risk of PTLD in children with Tac at discharge was significantly higher than that in children with CsA [1]. The use of MMF has not been associated with an increased risk of PTLD development [3739]. The effect of the proliferation signal inhibitors (PSIs), sirolimus and EVL, on PTLD development is not yet clear $[38,39]$. These drugs might be associated with reduced PTLD development because they display an inhibitory effect on PTLD derived cells in vitro and in vivo in an animal model $[40,41]$.

4.5. Cytokine Gene Polymorphisms. Cytokine gene polymorphisms may also influence the frequency of PTLD via cytokine production. Low interferon- $\gamma$ production may increase the risk in liver and kidney transplanted patients $[16,42]$.

\section{Clinical Presentation of B-PTLD}

As PTLD has a wide spectrum of disease manifestations, ranging from a benign plasmacytic hyperplasia and infectious mononucleosis-like PTLD to an aggressive monoclonal lymphoma, the clinical presentation of PTLD is highly variable and depends on the location of the disease. Often, it can present solely as tonsillar or adenoidal hypertrophy, as a single enlarged lymph node,or as an invasive mass in the abdomen, thorax, or anywhere that lymphoid tissue resides. It can affect nonlymphoid organs, including the allografts. The degree of disease also ranges from an isolated abnormal lymphadenopathy to fulminant systemic disease, often with comorbid infection. Fever (50\%), malaise, lymphadenopathy $(30 \%)$, and splenomegaly are common, and PTLD can masquerade as many different common childhood illnesses. When beginning in the tonsils and adenoids, the symptoms are of an exudative tonsillitis, upper respiratory infections, or infectious mononucleosis. Sometimes they show only noisy breathing, new onset of snoring, or changes in voice. Rapid growth of the tumor can lead to upper airway obstruction which sometimes requires tracheostomy. Most of pediatric transplant recipients have such otolaryngologic presentation $[43,44]$ associated with the early nondestructive form of PTLD with massive plasmacytic hyperplasia.

In a multi-institutional study [11] of 1184 pediatric primary HTx between 1993 and 2002 at 19 institutions in PHTS, in which early non-destructive lesions were excluded, 56 (5\%) developed PTLD. Almost half of patients had a single site of disease (27 patients, $48 \%$ ). The most common sites were gastrointestinal tract (22 patients, 39\%) (as with the present case), lung or airways (14 patients, $25 \%$ ), and cervical adenopathy (ten patients, 18\%). Central nervous system disease occurred in only two patients (3.6\%). Gastrointestinal 
symptoms included diarrhea in 11, vomiting in six, anorexia in three, and abdominal pain lower gastrointestinal bleeding each in one case.

An abdominal presentation may include pain, vomiting, nausea, diarrhea (sometimes bloody), weight loss, a palpable mass, or hepatosplenomegaly. In some patients, the initial presentation is intestinal obstruction or perforation (15\%). Tai et al. [45] reported an increased risk of mortality in pediatric PTLD patients with abdominal involvement. In this series, eight patients had abdominal involvement; four required surgical interventions because of intussusception and bowel perforation $(n=1)$, bowel perforation $(n=2)$, or tumor debunking $(n=1)$. Four of the eight patients died from complications related to PTLD.

If the lung is involved, cough, chest pain, breathlessness, or simply a lower respiratory illness that does no clear may be presenting symptom. Central nervous system involvement is less frequent, as previously described [11].

The most fulminant, and fortunately rarest form presents like acute infectious mononucleosis and progresses to a clinical picture resembling septic shock, with tumor involvement of all organs, multisystem organ failure, and disseminated intravascular coagulation $[46,47]$. Sometimes, this presentation coincides with an allograft rejection episode, with fever, tachycardia, lethargy, and flu-like syndrome. The patient may have a gallop rhythm and enlarged liver. Caution is needed in the differential diagnosis of this form of PTLD as treatment with added immunosuppression could prove fatal.

The early peak of PTLD presentation occurs within the first month after HTx and is usually associated with primary EBV infection. On the other hand, late onset (longer than 2 years after HTx) PTLD usually is not related to pretransplant EBV status in the recipient, and many late tumors may actually be EBV negative [47]. Primary EBV infection possibly causes more severe disease than EBV reactivation. It was suggested that a high carrying load after HTx might be more important than implantation of an EBV-positive organ into an EBV naive recipient for predicting future PTLD [33] as with the present case. EBV reinfection (secondary primary infection) displays more aggressive disease than EBV reactivation in HSCT [48]. Bone marrow involvement may present with new onset or persistent reduction in white and red blood cell counts. Hemophagocytosis is occasionally observed in EBV-related T-PTLD $[49,50]$ as well as in BPTLD $[49,51]$. From these reasons, bone marrow biopsy is important to manage PTLD. Polymorphic PTLD may present with features overlapping early lesions and monomorphic PTLD. As a result of the variability of presentation, clinicians should have a suspicion of PTLD in any patient with a history of transplantation.

The use of rituximab in combination with chemotherapy has been reported to be effective. However, B-PTLDs are sometimes associated with life-threatening complications, such as intestinal perforation and cardiogenic shock probably due to cytokine/toxin release syndrome after chemotherapy combined with rituximab as with the present case.

5.1. Probability of Survival following PTLD Diagnosis. In a previous PHTS study [11], the probability of survival was $83 \%$
$(72 \%-94 \%)$ at 6 months, $75 \%(63 \%-87 \%)$ at 1 year, and $68 \%$ $(55 \%-81 \%)$ at 3 years after diagnosis. Most deaths occurred in the first 2 years after diagnosis with low risk of death beyond that time. By contrast with treatment of malignant lymphoma in the nontransplant setting, treatment of PTLD includes weaning of immunosuppression and the subsequent risk of rejection. Success, therefore, requires complete response of the disease as well as prevention of graft loss from acute and chronic rejection. For this reason, they also analyzed event-free survival, which was defined as probability of freedom from death, graft loss, or PTLD relapse. Relapse was included since it represents failure of first-line therapy and has generally been associated with poor outcomes [11]. Eventfree survival was $76 \%(64 \%-87 \%)$ at 6 months, $73 \%(61 \%-$ $86 \%)$ at 1 year, $61 \%(46 \%-75 \%)$ at 3 years, and $56 \%(41 \%-$ $72 \%)$ at 5 years after diagnosis. A $15 \%$ difference in eventfree survival at 3 years was noted between polymorphic and monomorphic diseases but was not significant $(P=0.235)$, and the confidence intervals for the survival estimates were very broad at this time because of the small numbers of patients (monomorphic disease 68\% [8,45-92], polymorphic disease $53 \%[8,34-72])$.

Risk factors for survival that were explored with univariate analyses were also evaluated in this study [11]. None were identified as being significant predictors of patient survival, including several variables that have previously been thought to affect PTLD outcomes. Examples include number of sites of disease (single versus multiple), use of intravenous immunoglobulin products, use of antiviral agents (aciclovir or ganciclovir), initial reduction of immunosuppression (discontinuation versus reduction), and histology (monomorphic versus polymorphic). In the small subgroup with monomorphic disease, patients receiving chemotherapy as first-line treatment had slightly better 2 -year survival (75\%, 95\% CI 45-100) than those receiving reduced immunosuppression (57\%, 21-94), but this trend was not significant $(P=$ $0.329)$; the analysis comprised only 18 patients. The outcome for the monomorphic group did not differ between those with early onset disease ( $<3$ years posttransplantation) and those with late-onset disease $(P=0.26)$ [11].

5.2. Cause of Death after PTLD. Allograft failure from rejection or transplant coronary arterial vasculopathy accounted for approximately one half of the mortality associated with a diagnosis of PTLD. Indeed, a multi-institutional study [11] showed that 16 of 56 pediatric patients suffering from PTLD after HTx, died (28\%), mostly with progressive disease or from cardiac problems due to proven or presumed acute or chronic rejection after reduction of immunosuppression (seven from progressive PTLD, three from acute rejection, three from coronary artery disease, one from PTLD with acute rejection, one from PTLD with sudden death, and one from graft failure, unspecified). Therefore, close surveillance for rejection during and after treatment is essential, no matter what regimen is used to treat the PTLD [47]. 
TABLE 1: Staging of non-Hodgkin lymphoma (NHL) [8].

\begin{tabular}{|c|c|}
\hline Stage 1 & $\begin{array}{l}\text { NHL is limited to one lymph node group (e.g., neck, underarm, groin, etc.) above or below the diaphragm, or NHL is in an } \\
\text { organ or site other than the lymph nodes (extranodal) but has not spread to other organs or lymph nodes. }\end{array}$ \\
\hline Stage 2 & $\begin{array}{l}\text { NHL is limited to two lymph node groups on the same side of the diaphragm, or NHL is limited to one extranodal organ } \\
\text { and has spread to one or more lymph node groups on the same side of the diaphragm. }\end{array}$ \\
\hline Stage 3 & $\begin{array}{l}\text { NHL is in two lymph node groups, with/without partial involvement of an extranodal organ or site above and below the } \\
\text { diaphragm. }\end{array}$ \\
\hline Stage 4 & NHL is extensive (diffuse) in one organ or site, with/without NHL in distant lymph nodes. \\
\hline
\end{tabular}

\section{Diagnosis}

The timely and accurate diagnosis of PTLD is essential for early intervention. The diagnosis of PTLD should be based on histological examination of biopsy tissue $[3,52]$. There is no separate staging system for PTLD and it is currently staged using the same system as non-Hodgkin lymphoma (NHL) in the normal population. Staging of the disease should include CT or magnetic resonance imaging (MRI) of the abdomen and thorax and bone marrow aspiration. Additional investigations should be performed as indicated, for example, CT scan or MRI of the cranium and spinal cord or further gastrointestinal imaging.

6.1. Staging of PTLD (The Ann Arbor Staging System: Table 1). PTLD is currently staged using the same system as NHL and is categorized on the basis of tumor burden. The Ann Arbor Staging System is the most popular system for classifying NHL. The Ann Arbor Staging groups are as follows [8].

After an NHL patient has been assigned a stage, this categorization may be refined by adding the biologic grade of the disease, that is, "low," "intermediate," or "high" grade. Other descriptive terms-such as "bulky" versus "nonbulky" disease and the presence or absence of B symptoms-may be used to fully describe a particular case of lymphoma.

6.2. Additional Designations (Applicable to Any of the Stages of Hodgkin Disease (HD) or NHL). They are as follows:

(i) A-absent (no) symptoms;

(ii) B-presence of any of the following B symptoms: fever, drenching night sweats, unexplained weight loss of $10 \%$ or more within the last 6 months, and severe itching;

(iii) E-involvement of a single extranodal (other than the lymph nodes) site that directly adjoins or is next to the known nodal group;

(iv) $\mathrm{X}$-presence of "bulky" disease, that is, a nodal mass whose greatest dimension is more than 10 centimeters in size, and/or a widening of the mediastinum (middle chest) by more than one-third;

(v) CS - clinical stage as obtained by doctor's examinations and tests;

(vi) PS-pathological stage as obtained by exploratory laparotomy (surgery performed through an abdominal incision) with splenectomy (surgical removal of the spleen).
An example is Stage 2BX, high-grade NHL = NHL in which the disease is limited to two lymph node groups on the same side of the diaphragm (e.g., neck, underarm); the lymphoma is high-grade, with bulky disease and the presence of B symptoms.

6.3. Monitoring of EBV Viral Loads and sIL2R Level. Monitoring of EBV viral loads and sIL2R can be helpful for suspecting or predicting the development of PTLD in most cases, though not all cases. The presence of PTLD within the allograft itself may sometimes be mistaken for acute rejection and if there is diagnostic doubt, in situ hybridization for EBV encoded RNA, and PCR for VDJ heavy-chain rearrangements to determine clonality may be helpful $[53,54]$. Pertinent blood tests include complete blood counts, serum LDH levels, blood levels of EBV viral loads, and serum levels of EBV antibodies. Monitoring of sIL2R is also useful to evaluate response to treatment as well as to evaluate disease extent as with the present case.

6.4. Contrast-Enhanced CT Scan and MRI. Although chest $\mathrm{X}$-ray can pick up pulmonary nodules, effusion, and hilar adenopathy, CT scanning is more accurate to find thoracic and abdominal pathologic changes. Staging is assessed using the same system as NHL in the normal population [55]. Staging of the disease should include CT scan or MRI of the abdomen and thorax and bone marrow aspiration study.

6.5. ${ }^{67} \mathrm{Ga}$ Scintigraphy. High ${ }^{67} \mathrm{Ga}$ avidity is shown by diffuse large cell lymphomas including diffuse histiocytic lymphoma and poorly differentiated lymphocytic lymphoma [56] as with the present case. Similar ${ }^{67} \mathrm{Ga}$ avidity is shown by highgrade and intermediate-grade lymphomas including Burkitt's lymphoma. ${ }^{67} \mathrm{Ga}$ avidity in low-grade lymphomas seems to be low but this is currently under debate. For these reasons a ${ }^{67} \mathrm{Ga}$ scan is necessary before therapy in untreated patients in order to evaluate whether lymphoma is ${ }^{67} \mathrm{Ga}$ avid or not. If the ${ }^{67} \mathrm{Ga}$ scan is negative, it should not be repeated.

${ }^{67} \mathrm{Ga}$ can be employed (a) for evaluation of response to treatment: ${ }^{67} \mathrm{Ga}$ scintigraphy accurately assesses tumor viability in the presence of post therapy residual disease detected by conventional radiological tools such as CT or MRI; (b) as a prognostic indicator in the prediction of outcome; (c) for evaluation of disease extent. The accuracy of ${ }^{67} \mathrm{Ga}$ scan is not superior to that of CT or MRI in staging lymphomas at presentation; however, it may be useful prior 
to therapy as a reference for treatment monitoring. ${ }^{67} \mathrm{Ga}$ is more effective in restaging because of the frequent presence of anatomical distortions/alterations following treatment.

6.6. ${ }^{18}$ F-FDG-PET/CT. Development of imaging techniques such as ${ }^{18} \mathrm{~F}$-FDG-PET/CT has resulted in increased diagnostic accuracy and allowed clinicians to distinguish primary malignant lesions from benign areas as with the present case. ${ }^{18}$ F-FDG-PET has been reported to provide superior information on staging of NHL when compared with conventional CT scans. Graute et al. [57] reported that PET revealed the cause of nonspecific symptoms in 9 of 17 patients; there were 5 cases of lymphoproliferative disease (PTLD), 2 carcinomas, and 2 cases of infection. Thus, ${ }^{18} \mathrm{~F}-$ FDG-PET and PET/CT in HTx recipients with non-specific unexplained symptoms may offer diagnostic stratification for malignancy and infections with a high sensitivity and modest diagnostic accuracy. However, PET scans are not infallible, because low-grade lymphomas may not be FDG avid, and PET cannot distinguish between tumor and chronic inflammation.

${ }^{18}$ F-FDG-PET/CT has been reported to be useful for not only diagnosis of PTLD but also evaluation of response to treatment $[58,59]$. It must be noted that PET cannot distinguish between tumor and chronic inflammation. PET using the tracer ${ }^{18}$ F-FDG incorporates metabolic tumor function with anatomic localization. Tumor volumes by PET in lymphoma as well as solid tumors have been associated with clinical outcome in several studies [60-62] as with the present case.

6.7. Histological Examination of Biopsied Tissue. Excision biopsy is preferable and needle biopsy should only be performed where excision of affected tissue in not practicable. Fine needle aspiration and cytology has little role. In situ hybridization with the EBER-1 probe [63] which labels EBVencoded RNA in infected cells is the most reliable histologic stain for the presence of EBV and its use is recommended in all cases of suspected EBV disease [52]. Analysis of EBV latent proteins such as EBNA2 and LMP1, which are expressed in type III latent immunoblasts of monomorphic PTLD with the exception of PTLD with plasmacytic differentiation [64], is useful for identifying the type of latency. EBV-infected lymphocytes in early and polymorphic PTLDs represent a mixture of latencies II, III, and, in at least one-third of infected cells, latency 0 [65].

Flow cytometric analysis of immunophenotypical markers is also useful to determine the type of cellular infiltrates and genetic evaluation for clonality of the tumor. Clonality can be assessed using immunoglobulin heavy chain gene rearrangement and EBV termini assays [64]. Monoclonality of B-cell and T-cell lesions can be determined by flow cytometric analysis, when immunoglobulin light chain ( $j$ or $k$ ) restriction or loss of surface or cytoplasmic immunoglobulin and abnormal T-cell antigen expression or loss is identified, respectively [53]. Association between clonality and prognosis is controversial. A study of pediatric PTLD after solid organ transplantation reported that monoclonality did not
TABLE 2: Current WHO classification of PTLD (2008).

\begin{tabular}{l}
\hline (I) Early lesions \\
Plasmacytic hyperplasia \\
Infectious mononucleosis-like \\
(II) Polymorphic PTLD \\
(III) Monomorphic PTLD (classify according to lymphoma they \\
resemble) \\
B-cell neoplasms \\
Diffuse large B-cell lymphoma (DLBCL) \\
Burkitt lymphoma \\
Plasma cell myeloma \\
Plasmocytoma-like lesion \\
Other \\
T-cell neoplasms \\
Peripheral T-cell lymphoma, not otherwise specified \\
Hepato splenic lymphoma \\
Other \\
(IV) Classical Hodgkin lymphoma-type PTLD
\end{tabular}

imply poor prognosis [54]. For purposes of staging distant involvement, a bone marrow biopsy and lumbar puncture should be also performed.

6.8. Histopathological Classification of PTLD (Table 2). PTLD is a unifying name for several benign and malignant lymphoproliferative lesions. Due to their frequence and poor prognosis, an emphasis is given to lymphomas; nevertheless, PTLD is not a synonym for posttransplantation lymphoma. The previous, widely used WHO classification of PTLD was modified in 2008 (the current WHO classification can be seen in Table 1) [66-68]. Differences between the previous and the new WHO classification are few. There are two important changes: (1) many Burkitt-like lymphomas (according to the previous WHO classification) now fall into the new category of unclassifiable B-cell lymphoma, with intermediate features between DLBCL and Burkitt lymphoma; (2) Hodgkin lymphoma-like PTLD (according to the previous WHO classification), based on its immunophenotype, is currently considered DLBCL and belongs to the monomorphic PTLD group. These changes have an impact on the choice of treatment, which is different for each type of lymphoma [69]. follows.

The four subtypes of PTLD can be characterized as

(I) Early Lesions. Two histological patterns are described: (1) plasmacytic hyperplasia-the architecture of the involved tissue is generally retained, and the lymphoid tissue shows sheets of plasma cells with scattered EBV-positive large immunoblasts. (2) Infectious mononucleosis- (IM-) like PTLD-morphology is similar to IM; there is an expansion of 
the T-cell zone by immunoblasts and plasma cells. Cytologic atypia of early lesions is minimal $[69,70]$.

(II) Polymorphic PTLD. Histology shows effacement of underlying tissue architecture and a mixed infiltrate with small and medium-sized lymphocytes, immunoblasts, and plasma cells. High mitotic rate and atypical lymphoid cells may be observed. Early lesions and polymorphic PTLD are specific for transplanted patients. They mostly occur in childhood and are usually related to primary EBV infection. The other types of PTLD (see later) can also be diagnosed in immunocompetent individuals $[16,69,70]$.

(III) Monomorphic PTLD. B-cell PTLD is the most common form of PTLD.

Histology shows the destruction of underlying tissue architecture and malignant cytological features. There are four main categories. Diffuse largeB-cell lymphoma shows immunoblastic, centroblastic, or pleomorphic morphology. Burkitt lymphoma shows monomorphic cells with prominent apoptosis. Plasma cell myeloma and plasmocytomalike lesions contain sheets of mature plasma cells. The Tcell PTLD group includes T/Natural killer (NK)-cell lesions. Histology shows a wide range of morphological appearances depending upon the type of T-cell lymphoma $[16,69,70]$.

(IV) Classical Hodgkin Lymphoma-Type PTLD. This rare form of PTLD shows the histology of Hodgkin lymphoma in immunocompetent patients.

The mixed cellularity form is most frequent $[69,70]$.

PTLD is almost always of recipient origin following solid organ transplantation, whereas it may be donor-derived following hematopoietic stem cell transplantation. In Europe and in the USA approximately $85 \%$ of PTLDs are of B-cell origin, $80 \%$ of which is associated with EBV. The proportion of T-cell PTLD is 10\%-15\%; however, it may be as high as $40 \%$ in the Far East, due to the presence of human T-cell leukemia virus (HTLV-1). NK cell derived forms are extremely rare $[10,71]$.

\section{Basic Treatment of PTLD}

The treatment of PTLD is a complex task requiring special considerations. Therapeutic design requires the cooperation of an oncohematologist with experience in lymphoma therapy, a transplant specialist with experience in immunosuppression, and an infectionologist. Therapeutic decisions must be based on multiple factors: Ann Arbor staging and WHO classification [8], EBV status, patient performance, the transplanted organ, and the drugs used for immunosuppressive therapy. The transplanted organ and the lymphoproliferative disease must be both considered when establishing a therapeutic plan. In the case of crucial organs (liver, lung, and heart)-where the impairment of the transplanted organ can determine the fate of the patient-the protection of the graft is of utmost importance. Therefore, PTLD therapy must be planned individually.
7.1. Reduction in Immunosuppression with Frequent Echocardiography. The initial treatment in all patients with PTLD is to reduce immunosuppression in the hope that this will increase antitumor activity of cytotoxic T lymphocytes (CTLs) [3]. When patients are to receive chemotherapy, reduction in immunosuppression is a necessary component of treatment to minimize neutropenic sepsis. In EBV-driven PTLD, reducing immunosuppression allows host CTL function to be at least partially restored, hopefully resulting in an increase of EBV-specific CTLs and elimination of virally infected lymphocytes, including those which constitute the tumor.

As HTx recipients had a life-supporting graft, it is impossible to discontinue all immunosuppressive drugs. At first, any calcineurin inhibitor (CNI), such as Tac or CsA, is typically decreased over 4-6 weeks to a low level (around 50\%$75 \%$ of the normal therapeutic level) depending on the extent of disease, while the patient is observed closely for regression of tumor and also, equally importantly, for allograft rejection. A response to reduction in immunosuppression is usually seen within 2-4 weeks [52]. Reduction in immunosuppression alone leads to long-term disease remission in 40\%-86\% of cases of PTLD in pediatric transplant recipients [72-74]. This treatment can succeed in early lesion or polymorphic PTLD but is less successful in monomorphic types.

In a previous PHTS study, of the 31 patients with polymorphic disease treated with reduced immunosuppression as first-line therapy, 19 (61\%) developed rebound acute cellular rejection during the first 6 months after diagnosis of PTLD [11]. Median time to rebound rejection was 5 weeks (range 1-22 weeks). The diagnosis of acute rejection was confirmed by endomyocardial biopsy in all but three cases. Rebound rejection was slightly more common when immunosuppression was initially discontinued (ten of 14 cases, $71 \%$ ) rather than lowered (nine of $17,53 \%$ ), but this difference was not significant $(P=0.29)$. When all patients were included in the analysis (irrespective of histology), rebound rejection was more common if reduction or cessation of immunosuppression was used as first-line therapy (24 of 40, $60 \%$ ), compared with chemotherapy (five of $16,31 \% ; P=$ 0.032 ). In the same cohort, chronic rejection (post-transplant coronary artery disease) developed in ten patients after the diagnosis of PTLD, and two underwent retransplantation for this indication. PTLD did not recur after retransplantation [11].

As minimization of immunosuppression often causes allograft rejection, careful observation on allograft function is required. As it was reported that echocardiography was useful to diagnose cardiac allograft rejection in children [75] and an invasive examination such as endomyocardial biopsy is not favorable in patients with PTLD especially treated with EVL, frequent (once or twice weekly) echocardiography is recommended to monitor allograft rejection during treatment of PTLD, especially associated with life-threatening complication. If echocardiography showed signs of allograft rejection, such as an increase in LV wall thickness, mitral regurgitation, or pericardial effusion, a dose of immunosuppressive drugs should be increased or immunosuppressive regimen should be changed. 
After complete remission of the PTLD is achieved, the patient still requires immunosuppression to prevent the cardiac allograft. A narrow therapeutic window must be maintained to prevent allograft rejection and to prevent relapse of PTLD. As previously described, allograft failure from rejection and transplant coronary arterial vasculopathy accounted almost half of the mortality in patients with PTLD. The PSIs have both immunosuppressive and antiproliferative effects. Their activity is exerted through inhibition of the mammalian target of rapamycin (mTOR), such as sirolimus and EVL, resulting in inhibition of growth factor induced proliferation of lymphocytes, as well as other hematopoietic and nonhematopoietic cells of mesenchymal origin. They have also been reported to inhibit the growth of EBVtransformed B lymphocyte in vitro and in vivo [40, 41, 76]. mTOR-signaling pathways have been documented to be constitutively activated by lesional cells in PTLD [77]. PSIs might therefore be promising agents for preventing rejection and PTLD development [78]. CNI minimization with PSIs or conversion from CNIs to PSIs is currently being trialed, with the additional possibility of renal function preservation $[79,80]$. The use of PSIs has been reported in association with pediatric PTLD $[81,82]$. Further studies are needed for evaluation of the efficacy of PCIs. However, as with present case, PTLD also developed in children treated with EVL and further study should be needed.

7.2. Chemotherapy and Rituximab. Chemotherapy is commonly used when the reduction in immunosuppression fails to control the disease and as an initial therapy for aggressive, monoclonal PTLD, often late-onset PTLD. The most widely used cytostatic combination is CHOP (cyclophosphamide, adriamycin, vincristine, and prednisone) $[17,83$, 84]. Chemotherapy can, however, cause severe toxic and septic complications, increasing lethality. Therefore, chemotherapy is often combined with hematopoietic growth factors to reduce neutropenic sepsis. Low dose chemotherapy may decrease the occurrence of toxic complications but will lead to a higher relapse rate [46].

The PHTS study showed lower rejection rates when chemotherapy was used as primary therapy [11]. In the subgroup with monomorphic disease, patients receiving chemotherapy as first-line therapy had slightly better 2-year survival $(75 \%)$ than those receiving reduced immunosuppression (57\%). Of the patients with polymorphic PTLD treated with reduced immunosuppression as first-line therapy, 61\% developed rebound acute cellular rejection during the first 6 months after diagnosis of PTLD. A fine balance between management of PTLD and preserving allograft from rejection is therefore highly important.

Rituximab is a chimeric mouse/human monoclonal antibody that binds to the CD20 antigen on the surface of most normal and malignant B cells and can induce lysis of CD20 antigen-positive cells. Therefore, the use of monoclonal antibodies against CD20 antigen is a logical therapeutic approach against B-PTLD. In one series of pediatric transplant recipients with PTLD, 11 of 13 children had complete resolution of the disease after administration of 1-4 doses of rituximab (375 mg/m $\mathrm{m}^{2}$ per dose) alone [85]. Remission rates with the use of rituximab, some with concomitant reduction in immunosuppression, have been reported as $44 \%-87 \%$ [86-88].

Rituximab has been widely used for treatment of B-cell lymphoma as an adjunct to chemotherapy in non-transplant populations and reported to be effective compared to treatment with standard chemotherapy alone [89-92]. Rituximab may allow lowering of chemotherapy doses in attempt to reduce toxicity, particularly in pediatric patients [85]. A large retrospective study by Elstrom et al. [84] reported the efficacy of CHOP chemotherapy with or without rituximab. In this study, 23 patients received chemotherapy (CHOP, $n=10$; rituximab plus CHOP (R-CHOP), $n=9$; other regimens, $n=$ 4) with an overall response rate of $74 \%$. Currently, rituximab plus combination chemotherapy, such as R-CHOP, may be a more suitable option for the treatment of PTLD because of concurrently maintaining immunosuppression for allograft protection. However, caution is needed regarding the toxicity of combination chemotherapy [84]. In a study of six pediatric transplant recipients with PTLD who were treated with rituximab and reduced dose chemotherapy, five showed a complete response with only limited toxicity [93]. In pediatric transplant recipients treated with low-dose chemotherapy, consisting of cyclophosphamide and PRD, 67\% failurefree survival (without PTLD and with functioning original allograft) after failure with front-line therapy was achieved [46]. It may be effective by simultaneously controlling PTLD, preventing or treating allograft rejection, and minimizing treatment-related mortality. Long-term follow-up studies, however, are needed for full evaluation of the use of rituximab because it does not restore the CTL activity essential for longterm EBV control and relapse may therefore be a problem.

Despite these treatments, the overall lethality of PTLD after solid organ transplantation is around 50\% [3]. Different types of PTLD respond differently to treatment, and their prognosis is variable. The prognosis of early PTLD is better, whereas that of late forms is poor. Also, $40 \%-50 \%$ of kidney and heart transplanted patients with high grade lymphomas die in the first year; 5-year patient survival is around $30 \%$ for high-grade lymphomas. The prognosis of EBV-negative lymphomas is worse $[17,18]$.

\section{Life-Threatening Complications Associated with Chemotherapy Combined with Rituximab}

8.1. Gastrointestinal Perforation. Gastrointestinal involvement is one of the important determinants of the subsequent clinical course of lymphoma as with the present case and has a high mortality rate as described by Tai et al. [45]. Free gastrointestinal perforation is a potentially lethal complication of PTLD as well as NHL (as with the present case). The perforation can occur at any point in the gastrointestinal tract including the stomach, jejunum, ileum, and colon. Intestinal perforation due to intestinal lymphoma has been reported by many observers $[94,95]$ as well as PTLD $[4,45,96]$ as with the present case. 
In the last decade, rituximab has been used for the treatment of PTLD with promising results and significant reduction in toxicity and treatment-associated mortality when compared with chemotherapy [3]. However, the use of this recombinant chimeric anti-CD20 monoclonal antibody has been associated with spontaneous gastrointestinal perforation [4, 97-100]. In 2006, a warning was sent out by Roche Pharmaceuticals about the risk of intestinal perforation with rituximab. The company identified 37 cases of gastrointestinal perforations based on approximately 730,000 cumulative patient exposures, resulting in 4 fatalities. In addition, a pooled analysis of clinical trials in patients with non-Hodgkin lymphoma revealed a higher frequency of gastrointestinal perforation with rituximab and chemotherapy versus chemotherapy alone $(0.38 \%$ and $0.15 \%$, resp.) [101, 102]. Rituximab attacks the CD20 receptors causing lysis of the tumor cells. Postulated mechanisms of the tumoricidal activity of rituximab include complement-dependent cytotoxicity, antibody-dependent cell-mediated cytotoxicity and induction of apoptosis [103]. The first report of intestinal perforation after rituximab infusion in a patient with PTLD was by Kollmar et al. [4]. He suggested that the aggressive tumor lysis response may be related to the high CD20 expression of PTLD tumors. Nevertheless, in some cases [98] the tumor cells stained weakly positive for CD20 showing that the response to rituximab in relation with the degree of expression of this marker is not an accurate predictor of response.

As reported in previous studies free intestinal perforations due to NHL may occur both spontaneously and after chemotherapy as with the present case. Systemic administration of chemotherapy may increase the possibility of perforation if gastrointestinal involvement is present. When lymphoma invades the gastrointestinal tract and is treated with effective chemotherapy, tumor necrosis with perforation is the potential complication [93]. This complication has been reported especially in elderly patients with gastrointestinal manifestations of PTLD, multiple sites of involvement and advanced disease $[4,97,98]$. The prognosis in patients with advanced disease is poor [104]; however, in the setting of localized disease the treatment of PTLD may result in involution of gastrointestinal lesions.

Diagnosis is often delayed because steroids often mute symptoms. Small bowel and colon perforations may go unrecognized until the development of acute abdomen. In these cases the presence of free fluid on ultrasonography may be due to intestinal perforation despite normal plain abdominal X-ray findings. Therefore early diagnosis and treatment are important to improve the prognosis of bowel perforation in patients with PTLD as well as NHL.

Surgical treatment for small intestinal and colon lymphoma for localized lesions is resection of the affected segment and mesentery and primary anastomosis. For synchronous lymphoma, curative resection may not be performed effectively. In the treatment of a high-grade intestinal NHL, a multimodality approach is superior to surgery or chemotherapy alone. Perforated intestinal lymphomas usually are of a higher stage and have a poor prognosis. It was reported that surgery for perforated intestinal lymphomas had high mortality due to sepsis and high reoperation rate $[94,95]$. Therefore, in transplant patients who require immunosuppressive drugs, especially in patients treated with PSIs which prevent wound healing, jejunostomy or colostomy should be made as with the present case.

Although data regarding PTLD risk with use of anti-IL2R antibodies (basiliximab and daclizumab) have shown conflicting results, thymoglobulin was reported to be associated with a significantly increased risk of PTLD after renal transplantation, but alemtuzumab, basiliximab, and daclizumab, which trended toward a protective effect, were not [105]. And rabbit or horse anti-thymocyte globuline (ATG) cannot be used repeatedly. Therefore, anti-IL2R antibody is useful to withdraw or minimize other immunosuppressive drugs for about 10 days after surgery until infectious peritonitis due to gastrointestinal perforation is cured or until the patient can take oral medicine as with the present case. If the patients cannot take oral medicine for a long time after surgery, intravenous CNI infusion with relative high dose PRD is given to protect the allograft as with the present case. As additional chemotherapy after a surgery may have a risk for a leakage and another intestinal perforation, the stoma should be took down after a few courses of chemotherapy are finished and tumor burden is decreased. PSIs can be started a few weeks after giving a few courses of chemotherapy as with the present case or taking down the stoma (unpublished adult case).

8.2. Cardiac Graft Dysfunction Associated with Chemotherapy and Rituximab. Cardiac dysfunctions associated with chemotherapy and rituximab were also reported by many investigators, as well as PTLDs in cardiac allograft which are rare but have a high mortality rate [104] as with the present case.

Firstly, several drugs for chemotherapy have cardiac toxicity to lead ventricular dysfunction and DCM. Therefore, in HTx patients with PTLD, a reduced dose of such drugs is recommended and rituximab is useful to reduce a dose of such drugs [106-108].

Secondly, systemic inflammatory response syndrome (SIRS) associated with cytokine release syndrome (CRS) and disseminated intravascular coagulation occurring within 24 hour of administering rituximab have rarely been reported in the literature $[87,108-112]$. The pathophysiology of this reaction is attributed to release of cytokines generally after the first administration of rituximab. If uncontrolled, this can lead to SIRS. It carries a very high morbidity and case fatality rate. Cases of CRS reported within the literature are of patients with a very high tumor burden leading to a catastrophic cascade of events. CRS is characterized by an increase of inflammatory cytokines such as IFN- $\gamma$, IL-8, and TNF- $\alpha$ occurring about $90 \mathrm{~min}$ after the first infusion [109]. In severe cases of CRS after rituximab, a 5 to 10 fold increase in liver enzymes, elevation of D-dimer, LDH, and prolongation of prothombin time are also commonly observed [110]. Although this is based on case reports and causality is not established, it seems that fatal CRS/SIRS appear to occur in patients with a very high tumor burden, 
as noted in the present case as well. Caution needs to be exercised specifically in this subset of patients. For example, if patients develop similar but less severe reactions, a reduced dose and/or pre-treatment prior to rituximab infusions may be used to reduce the number and severity of reactions with subsequent doses [111].

Thirdly, cytokine or toxin release from the apoptotic tumor cells and a higher tumor burden as well as intestinal perforation may lead to left ventricular dysfunction [110]. Cytokine release from the rapid tumor lysis by chemotherapy and rituximab and a higher tumor burden may develop CRS to SIRS resulting in cardiac dysfunction. The rapid turnover of tumor cells in such cases may add markedly to the cytokine milieu leading to a catastrophic cascade of events [111].

Although to the best of our knowledge there has been no report of plasma exchange (PE) in patients with late CRS after treatment of rituximab and chemotherapy, PE seemed to be useful to reduce serum cytokine and toxin and to restore cardiac function as with the present case. Millivard et al. reported a case with cardiogenic shock complicated successful treatment of refractory thrombotic thrombocytopenia purpura with rituximab [113]. In this case, PE improved cardiac function. Although the effect of PE on Chronic active EBV infection (CAEBV) has not been discussed up to date, Arai et al. reported a case in whom PE decreased the levels of cytokines and had effects on hemophagocytotic syndrome as well as on chronic active EBV infection. These results also indicated that depletion of cytokines was effective for control of the disease [114]. PE has been reported to be effective to save patients with an associated respiratory failure and hemodynamic shock due to pH1N1 influenza [115]. In patients with cardiac allograft dysfunction after chemotherapy and rituximab, PE seemed to be effective to save patient if there is no evidence of acute allograft rejection.

\section{Conclusion}

PTLD remains a serious complication after pediatric HTx, resulting from immunosuppression for prevention of allograft rejection. Most cases are EBV related B-cell tumors. Primary EBV infection is highly associated with the development of PTLD in younger children. Treatment strategy is based on the reduction of immunosuppression, rituximab, or chemotherapy depending on the type of PTLD. As minimization of immunosuppression often causes death from allograft loss, frequent observation of the cardiac graft by echocardiography during treatment is needed. Finally, as characteristics of each children with B-PTLD are different from those of others, individual strategy might be required to manage PTLD, and good collaboration with pediatric cardiac transplant physician and surgeon, hematologist, gastrointestinal surgeons, pathologist, infection control team, and radiologist is essential to diagnose and treat pediatric patients suffering from PTLD, especially in patients associated with life-threatening complications.

\section{References}

[1] R. Kirk, A. I. Dipchand, L. B. Edwards et al., "Registry of the The Registry of the International Society for Heart and Lung Transplantation: Fifteenth Pediatric Heart Transplantation Report2012," http://www.jhltonline.org/.

[2] J. Stehlik, L. B. Edwards, A. Y. Kucheryvaya et al., "The Registry of the International Society for Heart and Lung Transplantation: 29th Official Adult Heart Transplant Report-2012," The Journal of Heart and Lung Transplantation, vol. 31, no. 10, pp. 1052-1064, 2012.

[3] G. Végso, M. Hajdu, and A. Sebestyén, "Lymphoproliferative disorders after solid organ transplantation-classification, incidence, risk factors, early detection and treatment options," Pathology and Oncology Research, vol. 17, no. 3, pp. 443-454, 2011.

[4] O. Kollmar, S. Becker, M. K. Schilling, and C. A. Maurer, "Intestinal lymphoma perforations as a consequence of highly effective anti-CD20 antibody therapy," Transplantation, vol. 73, no. 4, pp. 669-670, 2002.

[5] J. C. Byrd, J. K. Waselenko, T. J. Maneatis et al., "Rituximab therapy in hematologic malignancy patients with circulating blood tumor cells: association with increased infusion-related side effects and rapid blood tumor clearance," Journal of Clinical Oncology, vol. 17, no. 3, pp. 791-795, 1999.

[6] S.-J. Wu, W.-C. Chou, B.-S. Ko, and H.-F. Tien, "Severe pulmonary complications after initial treatment with rituximab for the Asian-variant of intravascular lymphoma," Haematologica, vol. 92, no. 1, pp. 141-142, 2007.

[7] P. M. Millward, N. Bandarenko, P. P. Chang et al., "Cardiogenic shock complicates successful treatment of refractory thrombotic thrombocytopenia purpura with rituximab," Transfusion, vol. 45, no. 9, pp. 1481-1486, 2005.

[8] J. O. Armitage, "Staging non-Hodgkin lymphoma," Ca-A Cancer Journal for Clinicians, vol. 55, no. 6, pp. 368-376, 2005.

[9] G. Opelz and B. Döhler, "Lymphomas after solid organ transplantation: a collaborative transplant study report," American Journal of Transplantation, vol. 4, no. 2, pp. 222-230, 2004.

[10] A. L. Taylor, R. Marcus, and J. A. Bradley, "Post-transplant lymphoproliferative disorders (PTLD) after solid organ transplantation," Critical Reviews in Oncology/Hematology, vol. 56, no. 1, pp. 155-167, 2005.

[11] S. A. Webber, D. C. Naftel, F. J. Fricker et al., "Lymphoproliferative disorders after paediatric heart transplantation: a multiinstitutional study," The Lancet, vol. 367, no. 9506, pp. 233-239, 2006.

[12] M. Ross, P. Kouretas, P. Gamberg et al., "Ten- and 20-year survivors of pediatric orthotopic heart transplantation," The Journal of Heart and Lung Transplantation, vol. 25, no. 3, pp. 261-270, 2006.

[13] S.-Z. Gao, S. V. Chaparro, M. Perlroth et al., "Post-transplantation lymphoproliferative disease in heart and heart-lung transplant recipients: 30-Year experience at Stanford University," The Journal of Heart and Lung Transplantation, vol. 22, no. 5, pp. 505-514, 2003.

[14] R. Chinnock, S. A. Webber, A. I. Dipchand et al., "A 16-year multi-institutional study of the Role of age and EBV status on PTLD incidence among pediatric heart transplant recipients," American Journal of Transplantation, vol. 12, pp. 3061-3068, 2012.

[15] C. Benden, L. B. Edwards, A. Y. Kucheryavaya et al., "The Registry of the International Society for Heart and Lung 
Transplantation: Fifteenth Pediatric Lung and Heart-Lung Transplantation Report-2012," The Journal of Heart and Lung Transplantation, vol. 31, no. 10, pp. 1087-1095, 2012.

[16] L. Tsao and E. D. Hsi, "The clinicopathologic spectrum of posttransplantation lymphoproliferative disorders," Archives of Pathology and Laboratory Medicine, vol. 131, no. 8, pp. 12091218, 2007.

[17] J. F. George, S. V. Pamboukian, J. A. Tallaj et al., "Balancing rejection and infection with respect to age, race, and gender: clues acquired from 17 years of cardiac transplantation data," The Journal of Heart and Lung Transplantation, vol. 29, no. 9, pp. 966-972, 2010.

[18] N. A. Bakker, G. W. Van Imhoff, E. A. M. Verschuuren, and W. J. Van Son, "Presentation and early detection of post-transplant lymphoproliferative disorder after solid organ transplantation," Transplant International, vol. 20, no. 3, pp. 207-218, 2007.

[19] A. W. Loren, D. L. Porter, E. A. Stadtmauer, and D. E. Tsai, "Post-transplant lymphoproliferative disorder: a review," Bone Marrow Transplantation, vol. 31, no. 3, pp. 145-155, 2003.

[20] J. F. George, D. O. Taylor, E. D. Blume et al., "Minimizing infection and rejection death: clues acquired from 19 years of multi-institutional cardiac transplantation data," The Journal of Heart and Lung Transplantation, vol. 30, no. 2, pp. 151-157, 2011.

[21] R. Kirk, L. B. Edwards, A. Y. Kucheryavaya et al., “The Registry of the International Society for Heart and Lung Transplantation: Thirteenth official pediatric heart transplantation report-2010," The Journal of Heart and Lung Transplantation, vol. 29, no. 10, pp. 1119-1128, 2010.

[22] C. Benden, P. Aurora, M. Burch et al., "Monitoring of EpsteinBarr viral load in pediatric heart and lung transplant recipients by real-time polymerase chain reaction," The Journal of Heart and Lung Transplantation, vol. 24, no. 12, pp. 2103-2108, 2005.

[23] S. Schubert, C. Renner, M. Hammer et al., "Relationship of immunosuppression to Epstein-Barr viral load and lymphoproliferative disease in pediatric heart transplant patients," The Journal of Heart and Lung Transplantation, vol. 27, no. 1, pp. 100105, 2008.

[24] S. Schubert, H. Abdul-Khaliq, H. B. Lehmkuhl et al., "Diagnosis and treatment of post-transplantation lymphoproliferative disorder in pediatric heart transplant patients," Pediatric Transplantation, vol. 13, no. 1, pp. 54-62, 2009.

[25] M. Green, M. G. Michaels, B. Z. Katz et al., "CMV-IVIG for prevention of Epstein Barr virus disease and posttransplant lymphoproliferative disease in pediatric liver transplant recipients," American Journal of Transplantation, vol. 6, no. 8, pp. 1906-1912, 2006.

[26] M. Green, J. Bueno, D. Rowe et al., "Predictive negative value of persistent low Epstein-Barr virus viral load after intestinal transplantation in children," Transplantation, vol. 70, no. 4, pp. 593-596, 2000.

[27] M. A. Bingler, B. Feingold, S. A. Miller et al., "Chronic high Epstein-Barr viral load state and risk for late-onset posttransplant lymphoproliferative disease/lymphoma in children," American Journal of Transplantation, vol. 8, no. 2, pp. 442-445, 2008.

[28] R. Scheenstra, E. A. M. Verschuuren, A. De Haan et al., "The value of prospective monitoring of Epstein-Barr virus DNA in blood samples of pediatric liver transplant recipients," Transplant Infectious Disease, vol. 6, no. 1, pp. 15-22, 2004.

[29] D. A. Axelrod, R. Holmes, S. E. Thomas, and J. C. Magee, "Limitations of EBV-PCR monitoring to detect EBV associated post-transplant lymphoproliferative disorder," Pediatric Transplantation, vol. 7, no. 3, pp. 223-227, 2003.

[30] L. J. Swinnen, M. R. Costanzo-Nordin, S. G. Fisher et al., "Increased incidence of lymphoproliferative disorder after immunosuppression with the monoclonal antibody OKT3 in cardiac-transplant recipients," New England Journal of Medicine, vol. 323 , no. 25 , pp. 1723-1728, 1990.

[31] J. D. Dayton, M. E. Richmond, R. G. Weintraub, A. T. Shipp, M. Orjuela, and L. J. Addonizio, "Role of immunosuppression regimen in post-transplant lymphoproliferative disorder in pediatric heart transplant patients," The Journal of Heart and Lung Transplantation, vol. 30, no. 4, pp. 420-425, 2011.

[32] R. J. Gajarski, E. D. Blume, S. Urschel et al., "Infection and malignancy after pediatric heart transplantation: the role of induction therapy," The Journal of Heart and Lung Transplantation, vol. 30, no. 3, pp. 299-308, 2011.

[33] C. Manlhiot, S. M. Pollock-BarZiv, C. Holmes et al., "Posttransplant lymphoproliferative disorder in pediatric heart transplant recipients," The Journal of Heart and Lung Transplantation, vol. 29, no. 6, pp. 648-657, 2010.

[34] K. L. Cox, L. S. Lawrence-Miyasaki, R. Garcia-Kennedy et al., "An increased incidence of Epstein-Barr virus infection and lymphoproliferative disorder in young children on FK506 after liver transplantation," Transplantation, vol. 59, no. 4, pp. 524$529,1995$.

[35] S. Cao, K. L. Cox, W. Berquist et al., "Long-term outcomes in pediatric liver recipients: comparison between cyclosporin A and tacrolimus," Pediatric Transplantation, vol. 3, no. 1, pp. 2226, 1999.

[36] B. S. Younes, S. V. McDiarmid, M. G. Martin et al., "The effect of immunosuppression on posttransplant lymphoproliferative disease in pediatric transplant patients," Transplantation, vol. 70, no. 1, pp. 94-99, 2000.

[37] V. R. Dharnidharka, P.-L. Ho, D. M. Stablein, W. E. Harmon, and A. H. Tejani, "Mycophenolate, tacrolimus and posttransplant lymphoproliferative disorder: a report of the North American Pediatric Renal Transplant Cooperative Study," Pediatric Transplantation, vol. 6, no. 5, pp. 396-399, 2002.

[38] S. Caillard, V. Dharnidharka, L. Agodoa, E. Bohen, and K. Abbott, "Posttransplant lymphoproliferative disorders after renal transplantation in the United States in era of modern immunosuppression," Transplantation, vol. 80, no. 9, pp. 12331243, 2005.

[39] B. D. Kahan, Y. K. Yakupoglu, L. Schoenberg et al., "Low incidence of malignancy among sirolimus/cyclosporine-treated renal transplant recipients," Transplantation, vol. 80, no. 6, pp. 749-758, 2005.

[40] M. Majewski, M. Korecka, P. Kossev et al., "The immunosuppressive macrolide RAD inhibits growth of human EpsteinBarr virus-transformed B lymphocytes in vitro and in vivo: a potential approach to prevention and treatment of posttransplant lymphoproliferative disorders," Proceedings of the National Academy of Sciences of the United States of America, vol. 97, no. 8, pp. 4285-4290, 2000.

[41] M. Majewski, M. Korecka, J. Joergensen et al., "Immunosuppressive tor kinase inhibitor everolimus (RAD) suppresses growth of cells derived from posttransplant lymphoproliferative disorder at allograft-protecting doses," Transplantation, vol. 75, no. 10, pp. 1710-1717, 2003. 
[42] M. A. Nalesnik, A. Zeevi, P. S. Randhawa et al., "Cytokine mRNA profiles in Epstein-Barr virus-associated post-transplant lymphoproliferative disorders," Clinical Transplantation, vol. 13, no. 1, pp. 39-44, 1999.

[43] N. Sculerati and M. Arriaga, "Otolaryngologic management of posttransplant lymphoproliferative disease in children," Annals of Otology, Rhinology and Laryngology, vol. 99, no. 6, pp. 445450, 1990

[44] S. D. Zangwill, D. T. Hsu, M. R. Kichuk et al., "Incidence and outcome of primary Epstein-Barr virus infection and lymphoproliferative disease in pediatric heart transplant recipients," The Journal of Heart and Lung Transplantation, vol. 17, no. 12, pp. 1161-1166, 1998.

[45] C. C. Tai, J. L. Curtis, J. R. Szmuszkovicz et al., "Abdominal involvement in pediatric heart and lung transplant recipients with posttransplant lymphoproliferative disease increases the risk of mortality," Journal of Pediatric Surgery, vol. 43, no. 12, pp. 2174-2177, 2008.

[46] T. G. Gross, J. C. Bucuvalas, J. R. Park et al., "Lowdose chemotherapy for Epstein-Barr virus-positive posttransplantation lymphoproliferative disease in children after solid organ transplantation," Journal of Clinical Oncology, vol. 23, no. 27, pp. 6481-6488, 2005.

[47] L. J. Addonizio and G. J. Boyle, "Posttransplant malignancy: risk, factors, incidence, diagnosis, treatment," in Pediatric Heart Transplantation, C. E. Canter and J. K. Kirklin, Eds., vol. 2, Elsevier, Philadelphia, Pa, USA, 2007.

[48] K. Kawa, A. Sawada, M. Koyama, and M. Inoue, "Epstein-Barr virus infection after unrelated cord blood transplantation: reactivation or reinfection?" International Journal of Hematology, vol. 85, no. 3, pp. 267-269, 2007.

[49] A. Karras, E. Thervet, and C. Legendre, "Hemophagocytic syndrome in renal transplant recipients: report of 17 cases and review of literature," Transplantation, vol. 77, no. 2, pp. 238-243, 2004.

[50] N. Awaya, A. Adachi, T. Mori et al., "Fulminant Epstein-Barr virus (EBV)-associated T-cell lymphoproliferative disorder with hemophagocytosis following autologous peripheral blood stem cell transplantation for relapsed angioimmunoblastic Tcell lymphoma," Leukemia Research, vol. 30, no. 8, pp. 10591062, 2006.

[51] H. Chisuwa, Y. Hashikura, Y. Nakazawa et al., "Fatal hemophagocytic syndrome after living-related liver transplantation," Transplantation, vol. 72, no. 11, pp. 1843-1846, 2001.

[52] M. Green, M. G. Michaels, S. A. Webber, D. Rowe, and J. Reyes, "The management of Epstein-Barr virus associated posttransplant lymphoproliferative disorders in pediatric solidorgan transplant recipients," Pediatric Transplantation, vol. 3, no. 4, pp. 271-281, 1999.

[53] D. M. Walling, L. A. Andritsos, W. Etienne et al., "Molecular markers of clonality and identity in Epstein-Barr virusassociated B-cell lymphoproliferative disease," Journal of Medical Virology, vol. 74, no. 1, pp. 94-101, 2004.

[54] W. A. Mourad, A. Tulabah, A. Al Sayed et al., "The impact of the World Health Organization classification and clonality assessment of posttransplant lymphoproliferative disorders on disease management," Archives of Pathology and Laboratory Medicine, vol. 130, no. 11, pp. 1649-1653, 2006.

[55] S. B. Murphy, "Classification, staging and end results of treatment of childhood non-Hodgkin's lymphomas: dissimilarities from lymphomas in adults," Seminars in Oncology, vol. 7, no. 3, pp. 332-339, 1980.
[56] C. Divgi, "Imaging: staging and evaluation of lymphoma using nuclear medicine," Seminars in Oncology, vol. 32, no. 1, pp. S11S18, 2005.

[57] V. Graute, N. Jansen, H. Sohn et al., "Diagnostic role of wholebody 18F-FDG positron emission tomography in patients with symptoms suspicious for malignancy after heart transplantation," The Journal of Heart and Lung Transplantation, vol. 31, pp. 958-966, 2012.

[58] C. von Falck, B. Maecker, E. Schirg et al., "Post transplant lymphoproliferative disease in pediatric solid organ transplant patients: a possible role for [18F]-FDG-PET(/CT) in initial staging and therapy monitoring," European Journal of Radiology, vol. 63, no. 3, pp. 427-435, 2007.

[59] E. Bianchi, M. Pascual, M. Nicod, A. B. Delaloye, and M. A. Duchosal, "Clinical usefulness of FDG-PET/CT scan imaging in the management of posttransplant lymphoproliferative disease," Transplantation, vol. 85, no. 5, pp. 707-712, 2008.

[60] M.-K. Song, J.-S. Chung, H.-J. Shin et al., "Clinical significance of metabolic tumor volume by PET/CT in stages II and III of diffuse large B cell lymphoma without extranodal site involvement," Annals of Hematology, vol. 91, pp. 697-703, 2012.

[61] D. E. Spratt, R. Diaz, J. McElmurray et al., "Impact of FDG $\mathrm{PET} / \mathrm{CT}$ on delineation of the gross tumor volume for radiation planning in non-small-cell lung cancer," Clinical Nuclear Medicine, vol. 35, no. 4, pp. 237-243, 2010.

[62] T. H. La, E. J. Filion, B. B. Turnbull et al., "Metabolic tumor volume predicts for recurrence and death in head-and-neck cancer," International Journal of Radiation Oncology Biology Physics, vol. 74, no. 5, pp. 1335-1341, 2009.

[63] P. S. Randhawa, R. Jaffe, A. J. Demetris et al., "Expression of Epstein-Barr virus-encoded small RNA (by the EBER1 gene) in liver specimens from transplant recipients with post-transplantation lymphoproliferative disease," New England Journal of Medicine, vol. 327, no. 24, pp. 1710-1714, 1992.

[64] H.-J. Delecluse, E. Kremmer, J.-P. Rouault, C. Cour, G. Bornkamm, and F. Berger, "The expression of Epstein-Barr virus latent proteins is related to the pathological features of post-transplant lymphoproliferative disorders," American Journal of Pathology, vol. 146, no. 5, pp. 1113-1120, 1995.

[65] R. Shaknovich, K. Basso, G. Bhagat et al., "Identification of rare Epstein-Barr virus infected memory B cells and plasma cells in non-monomorphic post-transplant lymphoproliferative disorders and the signature of viral signaling," Haematologica, vol. 91, no. 10, pp. 1313-1320, 2006.

[66] N. L. Harris, E. S. Jaffe, J. Diebold et al., "The World Health Organization classification of neoplastic diseases of the haematopoietic and lymphoid tissues: Report of the Clinical Advisory Committee Meeting, Airlie House, Virginia, November 1997," Histopathology, vol. 36, no. 1, pp. 69-87, 2000.

[67] N. L. Harris, S. H. Swerdlow, G. Frizzera et al., "Posttransplant lymphoproliferative disorders," in World Health Organization Classification of Tumors. Pathology and Genetics of Tumors of Haematopoietic and Lymphoid Tissue, E. S. Jaffe, N. L. Harris, H. Stein, and J. W. Vardiman, Eds., pp. 264-269, IARC Press, Lyon, France, 2001.

[68] S. H. Swerdlow, S. A. Webber, A. Chadburn et al., "Posttransplant lymphoproliferative disorders (PTLD)," in WHO Classification of Tumours of Haematopoietic and Lymphoid Tissue, pp. 343-350, IARC Press, Lyon, France, 2008.

[69] K. Mucha, B. Foroncewicz, B. Ziarkiewicz-Wróblewska, M. Krawczyk, J. Lerut, and L. Pczek, "Post-transplant lymphoproliferative disorder in view of the new WHO classification: 
a more rational approach to a protean disease?" Nephrology Dialysis Transplantation, vol. 25, no. 7, pp. 2089-2098, 2010.

[70] A. Parker, K. Bowles, J. A. Bradley et al., "Diagnosis of posttransplant lymphoproliferative disorder in solid organ transplant recipients-BCSH and BTS Guidelines," British Journal of Haematology, vol. 149, no. 5, pp. 675-692, 2010.

[71] Y. Hoshida, T. Li, Z. Dong et al., "Lymphoproliferative disorders in renal transplant patients in Japan," International Journal of Cancer, vol. 91, pp. 869-875, 2001.

[72] M. Benkerrou, A. Durandy, and A. Fischer, "Therapy for transplant-related lymphoproliferative diseases," Hematology/ Oncology Clinics of North America, vol. 7, no. 2, pp. 467-475, 1993.

[73] T. V. Cacciarelli, M. Green, R. Jaffe et al., "Management of posttransplant lymphoproliferative disease in pediatric liver transplant recipients receiving primary tacrolimus (FK506) therapy," Transplantation, vol. 66, no. 8, pp. 1047-1052, 1998.

[74] K. A. Newell, E. M. Alonso, P. F. Whitington et al., "Posttransplant lymphoproliferative disease in pediatric liver transplantation: interplay between primary Epstein-Barr virus infection and immunosuppression," Transplantation, vol. 62, no. 3, pp. 370-375, 1996.

[75] M. M. Boucek, C. M. Mathis, M. S. Kanakriyeh, D. D. Hodgkin, R. J. Boucek Jr., and L. L. Bailey, "Serial echocardiographic evaluation of cardiac graft rejection after infant heart transplantation," The Journal of Heart and Lung Transplantation, vol. 12, no. 5, pp. 824-831, 1993.

[76] R. R. Nepomuceno, C. E. Balatoni, Y. Natkunam, A. L. Snow, S. M. Krams, and O. M. Martinez, "Rapamycin inhibits the interleukin 10 signal transduction pathway and the growth of Epstein Barr virus B-cell lymphomas," Cancer Research, vol. 63, no. 15, pp. 4472-4480, 2003.

[77] M. El-Salem, P. N. Raghunath, M. Marzec et al., "Constitutive activation of mTOR signaling pathway in post-transplant lymphoproliferative disorders," Laboratory Investigation, vol. 87, no. 1, pp. 29-39, 2007.

[78] J. Pascual, "Post-transplant lymphoproliferative disorder-the potential of proliferation signal inhibitors," Nephrology Dialysis Transplantation, vol. 22, supplement 1, pp. i27-i35, 2007.

[79] J. A. Sánchez-Brotons, J. M. Sobrino-Márquez, E. Lage-Gallé et al., "Preliminary experience with conversion from calcineurin inhibitors to everolimus in cardiac transplantation maintenance therapy," Transplantation Proceedings, vol. 40, no. 9, pp. 30463048, 2008.

[80] A. O. Zuckermann and A. Z. Aliabadi, "Calcineurin-inhibitor minimization protocols in heart transplantation," Transplant International, vol. 22, no. 1, pp. 78-89, 2009.

[81] C. Jiménez-Rivera, Y. Avitzur, A. H. Fecteau, N. Jones, D. Grant, and V. L. Ng, "Sirolimus for pediatric liver transplant recipients with post-transplant lymphoproliferative disease and hepatoblastoma," Pediatric Transplantation, vol. 8, no. 3, pp. 243-248, 2004.

[82] S. Kusuki, Y. Hashii, N. Fukushima et al., "Pediatric posttransplant diffuse large B cell lymphoma after cardiac transplantation," International Journal of Hematology, vol. 89, no. 2, pp. 209-213, 2009.

[83] G. Muti, S. Cantoni, P. Oreste et al., "Post-transplant lymphoproliferative disorders: improved outcome after clinicopathologically tailored treatment," Haematologica, vol. 87, no. 1 , pp. $67-77,2002$.
[84] R. L. Elstrom, C. Andreadis, N. A. Aqui et al., "Treatment of PTLD with rituximab or chemotherapy," American Journal of Transplantation, vol. 6, no. 3, pp. 569-576, 2006.

[85] M. D. Pescovitz, "The use of rituximab, anti-CD20 monoclonal antibody, in pediatric transplantation," Pediatric Transplantation, vol. 8, no. 1, pp. 9-21, 2004.

[86] V. Ganne, N. Siddiqi, B. Kamaplath et al., "Humanized antiCD20 monoclonal antibody (Rituximab) treatment for posttransplant lymphoproliferative disorder," Clinical Transplantation, vol. 17, no. 5, pp. 417-422, 2003.

[87] S. Choquet, V. Leblond, R. Herbrecht et al., "Efficacy and safety of rituximab in B-cell post-transplantation lymphoproliferative disorders: results of a prospective multicenter phase 2 study," Blood, vol. 107, no. 8, pp. 3053-3057, 2006.

[88] A. H. Blaes, B. A. Peterson, N. Bartlett, D. L. Dunn, and V. A. Morrison, "Rituximab therapy is effective for posttransplant lymphoproliferative disorders after solid organ transplantation: results of a phase II trial," Cancer, vol. 104, no. 8, pp. 1661-1667, 2005.

[89] B. Coiffier, E. Lepage, J. Brière et al., "Chop chemotherapy plus rituximab compared with chop alone in elderly patients with diffuse large-B-cell lymphoma," New England Journal of Medicine, vol. 346, no. 4, pp. 235-242, 2002.

[90] N. Mounier, J. Briere, C. Gisselbrecht et al., "Rituximab plus CHOP (R-CHOP) overcomes bcl-2-associated resistance to chemotherapy in elderly patients with diffuse large B-cell lymphoma (DLBCL)," Blood, vol. 101, no. 11, pp. 4279-4284, 2003.

[91] R. U. Trappe, S. Choquet, P. Reinke et al., "Salvage therapy for relapsed posttransplant lymphoproliferative disorders (PTLD) with a second progression of PTLD after upfront chemotherapy: the role of single-agent rituximab," Transplantation, vol. 84, no. 12, pp. 1708-1712, 2007.

[92] K. Fu, D. D. Weisenburger, W. W. L. Choi et al., "Addition of rituximab to standard chemotherapy improves the survival of both the germinal center B-cell-like and non-germinal center B-cell-like subtypes of diffuse large B-cell lymphoma," Journal of Clinical Oncology, vol. 26, no. 28, pp. 4587-4594, 2008.

[93] M. Orjuela, T. G. Gross, Y.-K. Cheung, B. Alobeid, E. Morris, and M. S. Cairo, "A pilot study of chemoimmunotherapy (cyclophosphamide, prednisone, and rituximab) in patients with post-transplant lymphoproliferative disorder following solid organ transplantation," Clinical Cancer Research, vol. 9, no. 10, pp. 3945S-3952S, 2003.

[94] C. Ara, S. Coban, C. Kayaalp, S. Yilmaz, and V. Kirimlioglu, "Spontaneous intestinal perforation due to non-Hodgkin's lymphoma: evaluation of eight cases," Digestive Diseases and Sciences, vol. 52, no. 8, pp. 1752-1756, 2007.

[95] T. Yokota, Y. Yamada, Y. Murakami et al., "Abdominal crisis caused by perforation of ileal lymphoma," American Journal of Emergency Medicine, vol. 20, no. 2, pp. 136-137, 2002.

[96] D. Hoefer, H. Bonatti, G. Poelzl, W. Mark, H. Ott, and H. Antretter, "Cecal perforation as a rare initial manifestation of post-transplant lymphoproliferative disorder after cardiac transplantation," The Journal of Heart and Lung Transplantation, vol. 24, no. 4, pp. 505-506, 2005.

[97] E. Kutsch, P. Kreiger, D. Consolini, and K. N. Furuya, "Colonic perforation after rituximab treatment for post-transplant lymphoproliferative disorder (PTLD)," Journal of Pediatric Gastroenterology and Nutrition, vol. 56, no. 6, p. e41, 2013.

[98] A. Cornejo, M. Bohnenblust, C. Harris, and G. A. Abrahamian, "Intestinal perforation associated with rituximab therapy for 
post-transplant lymphoproliferative disorder after liver transplantation," Cancer Chemotherapy and Pharmacology, vol. 64, no. 4, pp. 857-860, 2009.

[99] Y.-C. Hsu, W.-C. Liao, H.-P. Wang, M. Yao, and J.-T. Lin, "Catastrophic gastrointestinal manifestations of post-transplant lymphoproliferative disorder," Digestive and Liver Disease, vol. 41, no. 3, pp. 238-241, 2009.

[100] "Rituximab: first report of intestinal perforations in an elderly patient: case report," Reactions, vol. 1, no. 901, pp. 14-15, 2002.

[101] "Rituximab: gastrointestinal perforation," Prescrire International, vol. 16, no. 91, p. 201, 2007.

[102] Health Canada, "Reports of bowel obstruction and gastrointestinal perforations with Rituxan," Rituximab, November 2006, http://www.hc-sc.gc.ca/.

[103] J. J. Ifthikharuddin, L. A. Mieles, J. D. Rosenblatt, C. K. Ryan, and D. M. Sahasrabudhe, "CD-20 expression in posttransplant lymphoproliferative disorders: treatment with rituximab," American Journal of Hematology, vol. 65, no. 2, pp. 171$173,2000$.

[104] V. Leblond, N. Dhedin, M.-F. M. Bruneel et al., "Identification of prognostic factors in 61 patients with posttransplantation lymphoproliferative disorders," Journal of Clinical Oncology, vol. 19, no. 3, pp. 772-778, 2001.

[105] A. D. Kirk, W. S. Cherikh, M. Ring et al., "Dissociation of depletional induction and posttransplant lymphoproliferative disease in kidney recipients treated with alemtuzumab," American Journal of Transplantation, vol. 7, no. 11, pp. 2619-2625, 2007.

[106] H. Khedmat and S. Taheri, "Heart allograft involvement by posttransplant lymphoproliferative disorders: report from the PTLD. Int survey," Experimental and Clinical Transplantation, vol. 9, no. 4, pp. 258-264, 2011.

[107] M. D. Pescovitz, "The use of rituximab, anti-CD20 monoclonal antibody, in pediatric transplantation," Pediatric Transplantation, vol. 8, no. 1, pp. 9-21, 2004.

[108] V. Ganne, N. Siddiqi, B. Kamaplath et al., "Humanized antiCD20 monoclonal antibody (Rituximab) treatment for posttransplant lymphoproliferative disorder," Clinical Transplantation, vol. 17, no. 5, pp. 417-422, 2003.

[109] H. S. Kulkarni and P. M. Kasi, "Rituximab and cytokine release syndrome," Case Reports in Oncology, vol. 5, pp. 134-140, 2012.

[110] G. Seifert, T. Reindl, S. Lobitz, K. Seeger, and G. Henze, "Fatal course after administration of rituximab in a boy with relapsed all: a case report and review of literature," Haematologica, vol. 91, no. 6, supplement, p. ECR23, 2006.

[111] J. C. Byrd, J. K. Waselenko, T. J. Maneatis et al., "Rituximab therapy in hematologic malignancy patients with circulating blood tumor cells: association with increased infusion-related side effects and rapid blood tumor clearance," Journal of Clinical Oncology, vol. 17, no. 3, pp. 791-795, 1999.

[112] S.-J. Wu, W.-C. Chou, B.-S. Ko, and H.-F. Tien, "Severe pulmonary complications after initial treatment with rituximab for the Asian-variant of intravascular lymphoma," Haematologica, vol. 92, no. 1, pp. 141-142, 2007.

[113] P. M. Millward, N. Bandarenko, P. P. Chang et al., "Cardiogenic shock complicates successful treatment of refractory thrombotic thrombocytopenia purpura with rituximab," Transfusion, vol. 45, no. 9, pp. 1481-1486, 2005.

[114] A. Arai, A. Nogami, K. Imadome et al., "Sequential monitoring of serum IL-6, TNF-a, and IFN-c levels in a CAEBV patient treated by plasma exchange and immunochemotherapy," International Journal of Hematology, vol. 96, no. 5, pp. 669-673, 2012.
[115] P. Patel, V. Nandwani, J. Vanchiere, S. A. Conrad, and L. K. Scott, "Use of therapeutic plasma exchange as a rescue therapy in 2009 pH1N1 influenza A-An associated respiratory failure and hemodynamic shock," Pediatric Critical Care Medicine, vol. 12, no. 2, pp. e87-e89, 2011. 


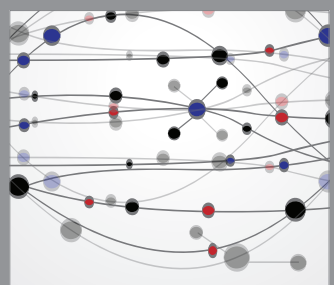

The Scientific World Journal
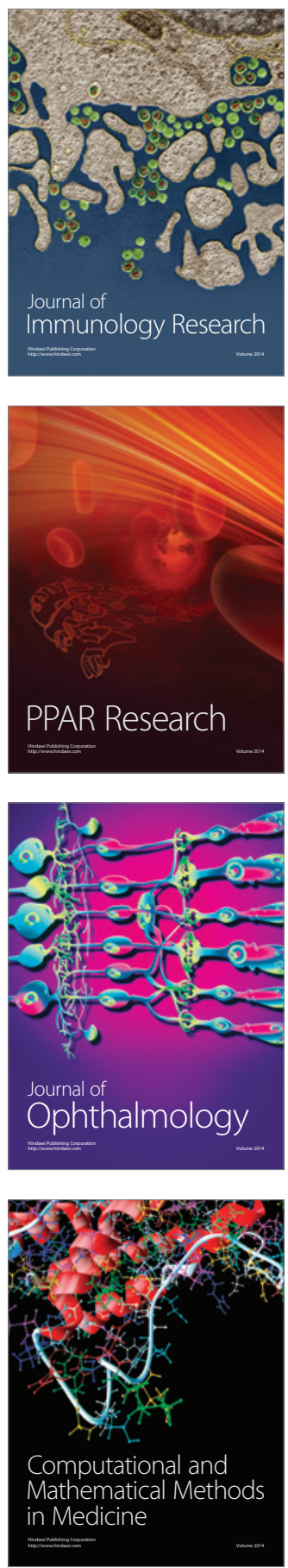

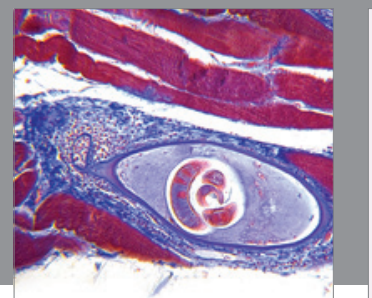

Gastroenterology

Research and Practice
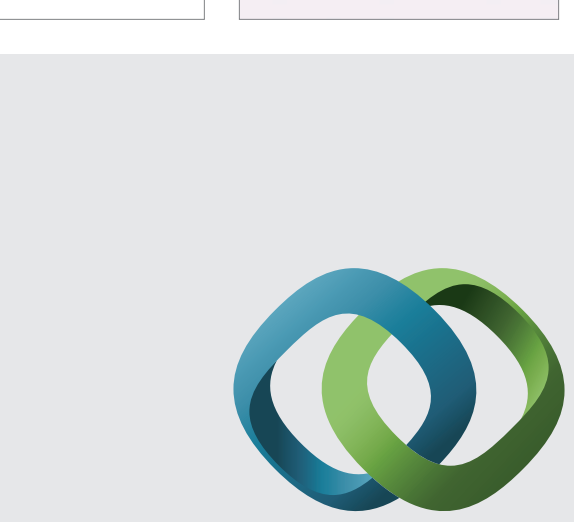

\section{Hindawi}

Submit your manuscripts at

http://www.hindawi.com
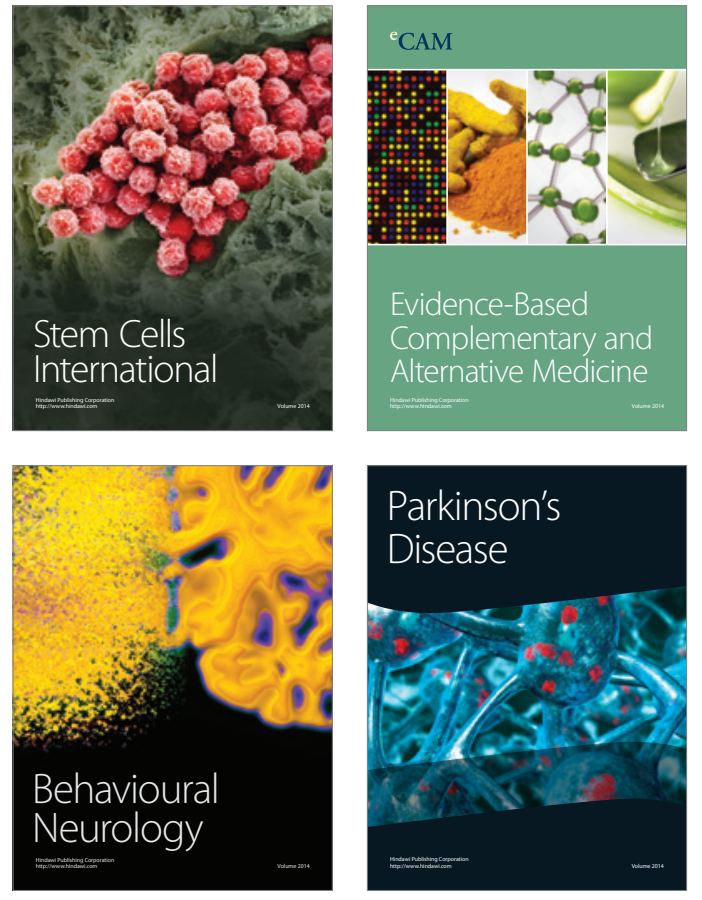
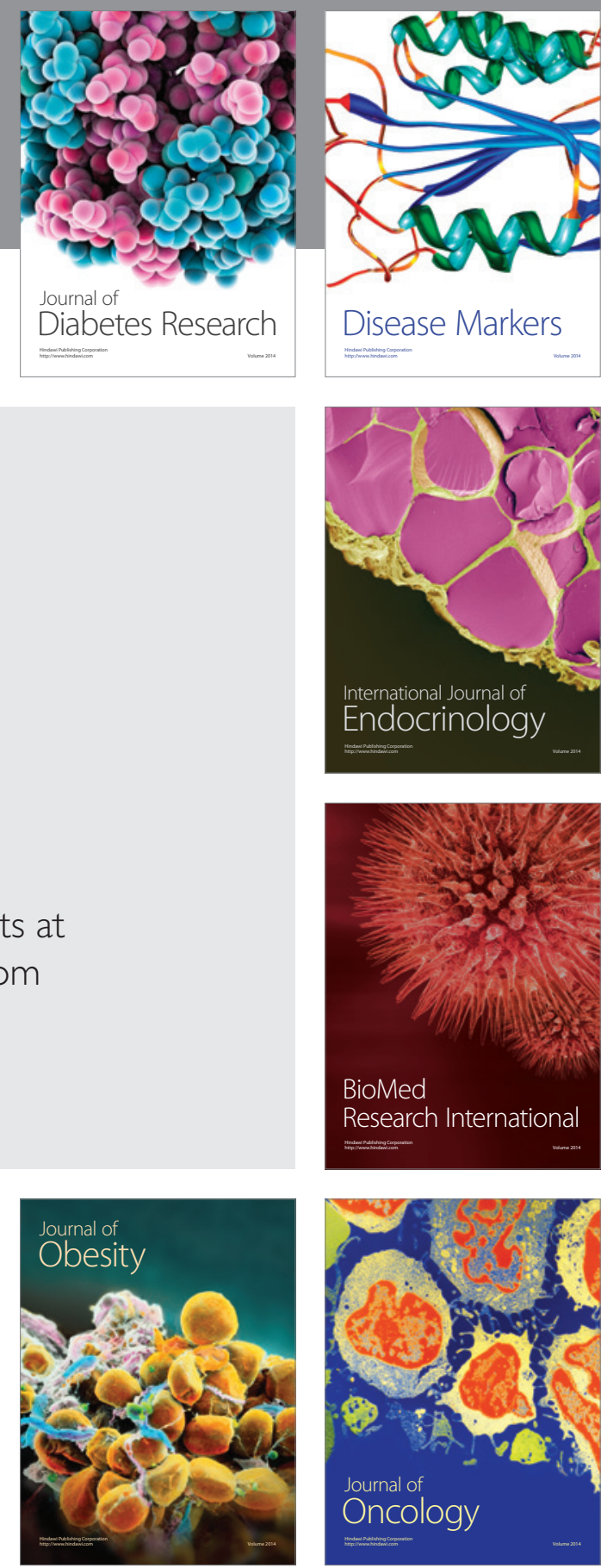

Disease Markers
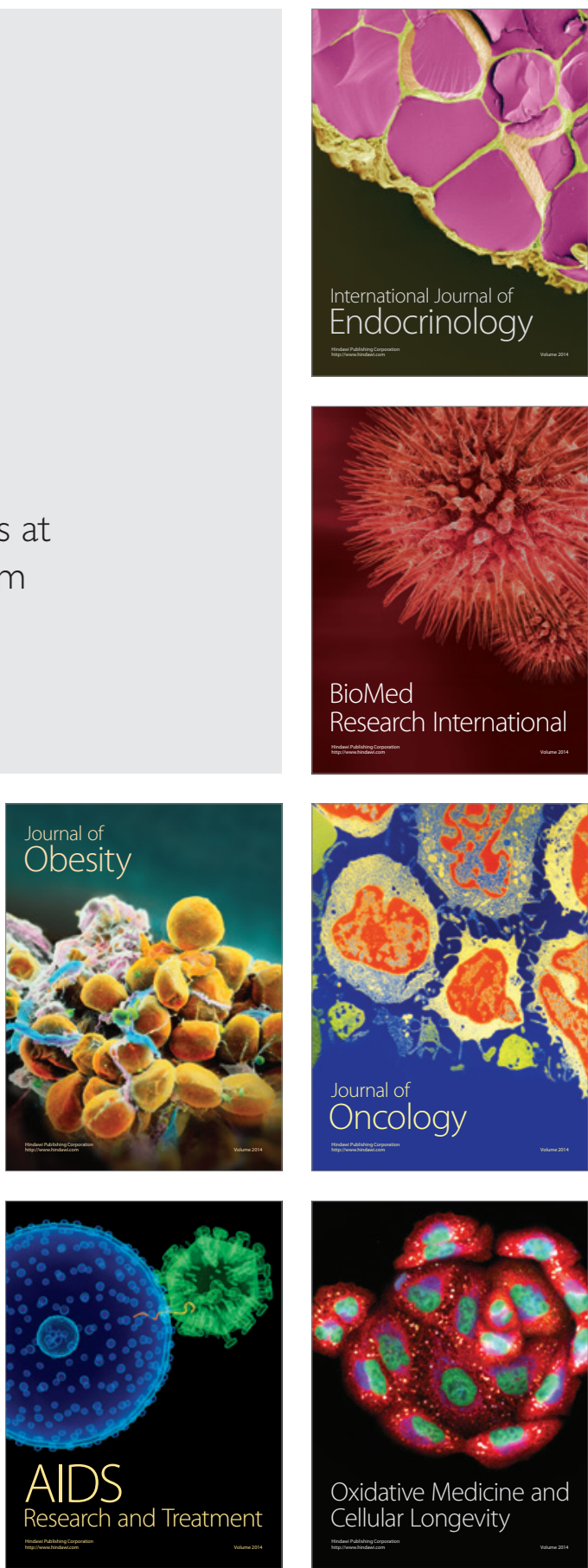